\title{
Thermo-elastic optical coherence tomography
}

\author{
Tianshi Wang, ${ }^{1, \dagger}$ Tom Pfeiffer, ${ }^{2, \dagger}$ Min Wu, ${ }^{1}$ Wolfgang Wieser, ${ }^{3}$ Gaetano Amenta, ${ }^{1}$ \\ Wolfgang Draxinger, ${ }^{2}$ Antonius F. W. van der Steen, ${ }^{1,4,5}$ Robert Huber, ${ }^{2}$ and Givs van Soest ${ }^{1, *}$ \\ ${ }^{1}$ Thorax Center, Erasmus University Medical Center, P. O. Box 2040, Rotterdam 3000 CA, The Netherlands \\ ${ }^{2}$ Institut für Biomedizinische Optik, Universität zu Lübeck, Peter-Monnik-Weg 4, 23562 Lübeck, Germany \\ ${ }^{3}$ Optores $\mathrm{GmbH}$, Gollierstr. 70, 80339 München, Germany \\ ${ }^{4}$ Shenzhen Institutes of Advanced Technology, Chinese Academy of Sciences, 518055 Shenzhen, China \\ ${ }^{5}$ Department of Imaging Science and Technology, Delft University of Technology, Postbus 5, Delft 2600 AA, The Netherlands \\ ${ }^{*}$ Corresponding author: g.vansoest@erasmusmc.nl
}

Received 19 July 2017; revised 8 August 2017; accepted 8 August 2017; posted 9 August 2017 (Doc. ID 302613); published 30 August 2017

\begin{abstract}
The absorption of nanosecond laser pulses induces rapid thermo-elastic deformation in tissue. A sub-micrometer scale displacement occurs within a few microseconds after the pulse arrival. In this Letter, we investigate the laserinduced thermo-elastic deformation using a $1.5 \mathrm{MHz}$ phase-sensitive optical coherence tomography (OCT) system. A displacement image can be reconstructed, which enables a new modality of phase-sensitive OCT, called thermo-elastic OCT. An analysis of the results shows that the optical absorption is a dominating factor for the displacement. Thermo-elastic OCT is capable of visualizing inclusions that do not appear on the structural OCT image, providing additional tissue type information. (๑) 2017 Optical Society of America
\end{abstract}

OCIS codes: (170.4500) Optical coherence tomography; (140.3538) Lasers, pulsed; (170.0110) Imaging systems; (170.3880) Medical and biological imaging.

https://doi.org/10.1364/OL.42.003466

Optical coherence tomography (OCT) provides images of biological samples with micrometer-order resolution [1-4]. It finds widespread application for medical diagnostic imaging, targeting especially the identification of diseased tissue in ophthalmology, cancer imaging, and cardiovascular imaging [5-11]. The conventional OCT image contrast is derived from elastic scattering and shows the internal structure of the sample. The determination of the tissue type in OCT images usually depends on the interpretation by the image reader. More accurate tissue type contrast may be achieved by new OCT-based imaging modalities, with sensitivity to other physical parameters than scattering alone [12-16].

Phase-sensitive OCT can detect tissue motion on nanometerto-micrometer length scales using the phase of the OCT signal. Depending on the nature of the excitation, different functional images can be reconstructed: a mechanical stimulus yields images of tissue elasticity [14,17-19], while continuous-wave laser illumination provides photo-thermal deformation images [20-25]. These techniques use complex and frequently large multimodal devices, or require long integration times (in the order of milliseconds). We aim for a method to detect optical absorbers, which can be robust in the presence of tissue motion, based on a small imaging probe (suitable for endoscopy) and short integration time (allowing fast imaging). In this Letter, we will use nanosecond laser pulses to induce rapid thermoelastic deformation in tissue, which can be detected by phase-sensitive OCT within a few microseconds.

Previous studies have described the thermo-elastic deformation in tissue following short-pulse laser absorption [26-30]. The absorbed energy first leads to a nonuniform temperature rise in the sample, which causes a rapid (nanosecond to microsecond depending on the illuminated volume and speed of sound) thermo-elastic expansion that sends out an elastic wave propagating through the tissue. A quasi-steady state is reached after the expansion. At that time, the sample reaches equilibrium, and the net force on the illuminated area is zero. During the subsequent relaxation phase, the stress slowly (milliseconds) decays to zero, as the temperature of the sample becomes uniform due to thermal diffusion. In a linear and isotropic material, the one-dimensional displacement $u(z, t)$ follows the following equation:

$$
\frac{\partial^{2} u}{\partial z^{2}}-\frac{1}{c_{L}^{2}} \frac{\partial^{2} u}{\partial t^{2}}=-\mu_{a} \mu_{\mathrm{att}} P \frac{\beta}{3 C_{p}} \frac{(1+\nu)}{\rho(1-\nu)} e^{-\mu_{\mathrm{att}} z} .
$$

Here, $\beta$ is the thermal expansion coefficient, $\rho$ is the density, $c_{L}$ is the speed of sound, $\mu_{a}$ and $\mu_{\text {att }}$ are the optical absorption and optical attenuation coefficient, respectively, $\nu$ is the Poisson's ratio, $C_{p}$ is the heat capacity, and $P$ is the optical fluence. The solution of the displacement in the quasi-steady state follows $[26,27,31]$ :

$$
\begin{aligned}
u(z, t)= & u_{0}\left\{\exp \left[-\mu_{\mathrm{att}} c_{L}\left(t-\frac{z}{c_{L}}\right)\right]\right. \\
& \left.+\exp \left[-\mu_{\mathrm{att}} c_{L}\left(t+\frac{z}{c_{L}}\right)\right]-2 \exp \left(-\mu_{\mathrm{att}} z\right)\right\} \\
\approx & -2 u_{0} \exp \left(-\mu_{\mathrm{att}} z\right),
\end{aligned}
$$

where 


$$
u_{0}=\frac{\mu_{a}}{\mu_{\text {att }}} \frac{(1+\nu)}{(1-\nu)} \frac{\beta}{C_{p}} \frac{P}{3 \rho} .
$$

The associated displacement $u$ in the homogenous sample caused by the thermo-elastic deformation can be extracted by calculating the phase shift $\phi$ before and after the irradiation following

$$
u=\phi \frac{\lambda_{0}}{4 \pi n},
$$

where $n$ is the refractive index of the sample, and $\lambda_{0}$ is the center wavelength of the OCT laser source [17].

In this Letter, we use phase-sensitive megahertz OCT (MHz-OCT) to investigate the associated displacement that is caused by the thermo-elastic deformation focusing on the optical absorption. We further introduce a new modality of phase-sensitive OCT, called thermo-elastic OCT (TE-OCT).

The TE-OCT prototype is composed of a wavelengthtunable pulsed laser and a phase-sensitive OCT system. The tunable laser (OPOTEK Vibrant B/355-II, U.S.) has pulse duration of $5 \mathrm{~ns}$ and a repetition rate of $10 \mathrm{~Hz}$. The excitation laser beam was coupled into a multimode fiber with a $400 \mu \mathrm{m}$ core diameter forming a beam spot on the sample with approximately $1.0 \mathrm{~mm}$ diameter. The laser was operated at 500 and $1200 \mathrm{~nm}$ wavelengths with a power of $2 \mathrm{~mJ}$ per pulse on the sample. The phase-sensitive OCT system is based on a $1.5 \mathrm{MHz}$ Fourier domain mode locked (FDML) laser [32-34] with a center wavelength of $1310 \mathrm{~nm}$, a sweep range of $90 \mathrm{~nm}$, an output power of $20 \mathrm{~mW}$ on the sample, a sensitivity of $102 \mathrm{~dB}$, an image depth of $4.0 \mathrm{~mm}$, and a phase stability of $0.5 \mathrm{~nm}$ in air. In the OCT engine, $90 \%$ of the laser power was sent to the sample, while $10 \%$ of the laser power was sent to the reference mirror. A $1.6 \mathrm{GHz}$ balanced photo receiver (Thorlabs, PDB480C-AC, U.S.) and a 4 GS/s 8 bit data acquisition board (Gage, COBRAMAX, U.S,) were used to record the interferogram. A mirror was used to calibrate the noise background and the wavenumber linearity using $5 \%$ power from the sample arm. The calibration mirror was blocked during data acquisition. A custom-made ball lens fiber probe focuses the OCT beam at $2 \mathrm{~mm}$ distance forming a $20 \mu \mathrm{m}$ beam waist. The ball lens probe and the multimode fiber were integrated into a forward-looking probe with an angle of 20 deg. Both the FDML laser and the tunable pulse laser were synchronized to the data acquisition card. A schematic of the TE-OCT prototype is shown in Fig. 1.

Elastic phantoms were prepared by dissolving $10 \%$ of polyvinyl alcohol (PVA) in water, clear or mixed with red ink (Canon, CLI-8, Japan) at volume ratios of $1 \%$ and $2.5 \%$. The

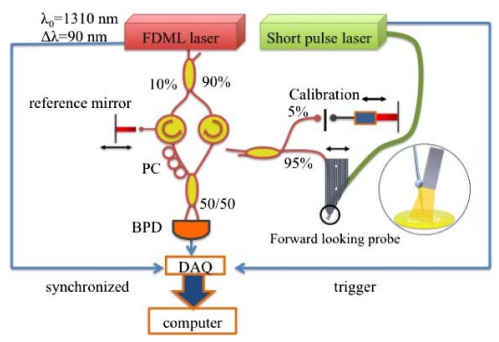

Fig. 1. Schematic diagram of TE-OCT prototype. FDML, Fourier domain mode locked; PC, polarization controller; BPD, balanced photodetector; DAQ, data acquisition card. white $(0 \%)$, pink $(1 \%)$, and red $(2.5 \%)$ phantoms were prepared with three freeze-thaw cycles [35]. Biological samples were collected from a healthy swine after sacrificing for a different experiment. The procedure was carried out in accordance with a protocol sanctioned by the animal welfare committee of Erasmus Medical Center.

For each laser pulse, successive and co-located A-lines were acquired and processed generating M-mode images [Figs. 2(a) and 2(b)]. Single TE-OCT A-line data were generated by averaging a group of four neighboring and co-located processed A-lines that were acquired after the deformation accumulation (6.5 $\mu \mathrm{s}$ after the irradiation). The averaging is to depress the phase noise and the tiny phase error between the master A-line and three buffered A-lines in each group. A two-dimensional (2D) TE-OCT image can further be created by linearly moving the forward-looking probe at a speed of $0.2 \mathrm{~mm} / \mathrm{s}$.

The thermo-elastic deformation was investigated in the red PVA phantom using a laser pulse operated at $500 \mathrm{~nm}$. The laser pulse power was attenuated by $20 \%$ to avoid phase wrap due to strong absorption. The results show that no deformation can be observed in the structural M-mode OCT image [Fig. 2(a)]. However, a depth-resolved phase shift can be detected right after the laser pulse irradiation [Fig. 2(b)]. The surface displacements were acquired in a PVA phantom mixed with red ink at volume ratios of $0 \%, 1.0 \%$, and $2.5 \%$. The results show that the thermo-elastic deformation stabilizes in less than $6.5 \mu$ s $(10$ A-lines). The temporal sampling is too low to capture the initial rapid expansion. Therefore, it was mainly the quasi-steady state that was observed using our system. The surface displacement at $20 \mu$ s after the irradiation was detected to be $115 \pm 14 \mathrm{~nm}$ in a $1.0 \%$ red PVA phantom and $197 \pm 19 \mathrm{~nm}$ in a $2.5 \%$ red PVA phantom. No significant surface displacement can be observed in the $0 \%$ red PVA phantom due to the negligible optical absorption [Fig. 2(c)]. Figure 2(d) shows the depth-resolved displacements that were acquired $6.5 \mu$ s after the pulse irradiation. The displacement decays with depth following an exponential function per Eq. (2). The attenuation coefficients were extracted by fitting the plots. The values are
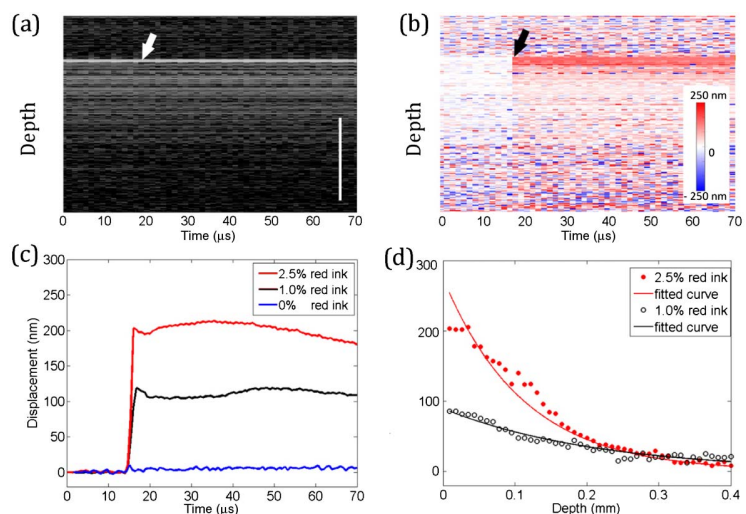

Fig. 2. (a) Collocated structural OCT A-lines (M-mode) of PVA phantoms. (b) Phase-resolved A-lines (M-mode) of the PVA phantom using a $500 \mathrm{~nm}$ pulse laser as an excitation source. The arrows indicate the laser pulse irradiation. (c) Surface displacement plots of PVA phantoms mixed with $0 \%, 1 \%$, and $2.5 \%$ red ink dye. (d) Exponential fitting of the depth-resolved displacement at $6.5 \mu$ s after irradiation. Scale bar in (a): $1.0 \mathrm{~mm}$. The displacement towards the probe is positive. 
$3.8 \mathrm{~mm}^{-1}\left(R^{2}=0.88\right)$ in the $1 \%$ red ink PVA phantom and $7.0 \mathrm{~mm}^{-1}\left(R^{2}=0.95\right)$ in the $2.5 \%$ red PVA phantom. Using Eq. (3), the absorption coefficient ratio between the two PVA phantoms is estimated to be $1: 2.98$. The small deviation from the expected ratio 1:2.5 may be due to the nonuniform dilution of the red ink in the PVA phantom. A contraction right after the rapid expansion can also be observed in Fig. 2(c). Previous studies have proven that the contraction is related to the stiffness of the sample [26].

A first 2D TE-OCT image consisting of 170 phase-resolved A-lines was acquired in a three-cycle PVA phantom with red ink dye injected at a depth of $200 \mu \mathrm{m}$ beneath the surface. The acquisition time was $17 \mathrm{~s}$ in total, limited by the repetition rate of the pulsed laser. The pulse laser was operated at a $500 \mathrm{~nm}$ wavelength with $100 \%$ power output. Figure 3(a) shows the structural OCT images of the phantom: no trace of the colored area can be observed. However, the TE-OCT image clearly shows the presence of the colored area with a promising contrast due to the strong deformation, as shown in Fig. 3(b). It can also be seen that the associated displacement is in both upward and downward directions centered on the inclusion. An upward (positive) displacement on the surface can also be observed which is due to the "pushing" of the colored area.

TE-OCT images (consisting of 250 phase-resolved A-lines each) were acquired in three different biological samples: a lipid target (swine fat), a healthy vessel wall (swine aorta artery wall), and a muscle (swine myocardium). The acquisition time was $25 \mathrm{~s}$ for each image. Figure 4 shows the detected surface displacements at two wavelengths. Due to the difference in optical absorption, the three tissue types show different deformation features. Lipid is strongly absorbing at $1200 \mathrm{~nm}$, compared to the artery wall and muscle, which leads to a stronger surface displacement. At $500 \mathrm{~nm}$ excitation, aorta artery wall, and heart muscle show a much stronger surface displacement due to the high optical absorption at this wavelength. Figure 5 shows the TE-OCT image of these three tissue types. Different structural features of these types of tissues can be observed in the structural OCT images. These features are normally the evidence for

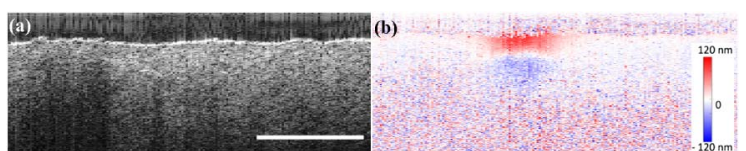

Fig. 3. TE-OCT image of a three-cycle PVA phantom with red ink dye injected at a depth of approximately $200 \mu \mathrm{m}$. (a) Structural OCT image and (b) phase-resolved TE-OCT image showing the colored area. Scale bar: $1.0 \mathrm{~mm}$; the displacement towards the probe is positive.

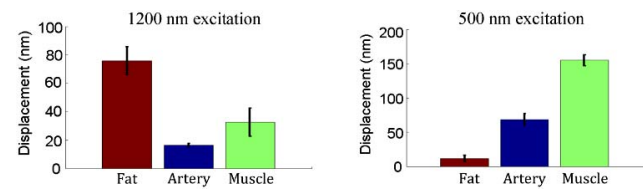

Fig. 4. Surface displacements of swine fat, aorta artery wall, and heart muscle using 1200 and $500 \mathrm{~nm}$ laser pulses for excitation. The bars represent standard deviation of the mean.

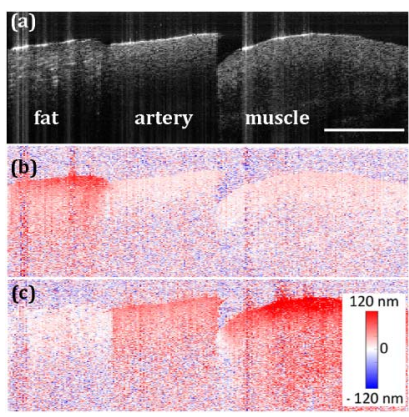

Fig. 5. (a) Typical structural OCT image of biological tissues, including swine fat, swine aorta artery wall, and swine heart muscle. (b, c) TE-OCT images of the samples using the short-pulse laser at $1200 \mathrm{~nm}$ in (b) and $500 \mathrm{~nm}$ in (c). Scale bar: $1.0 \mathrm{~mm}$. The displacement towards the probe is positive.

tissue identification using OCT. Figures 5(b) and 5(c) show the TE-OCT images using 500 and $1200 \mathrm{~nm}$ laser pulse excitation. It is much easier to identify swine fat in the TE-OCT image, as shown in Fig. 5(b). Furthermore, different tissue types can be highlighted in TE-OCT images by changing the excitation wavelength.

In summary, we studied short laser-pulse-induced thermoelastic deformation using a phase-sensitive MHz-OCT engine. Our phantom studies show that the associated displacement can be detected within a few microseconds. The deformation relies on the absorption of the laser pulse. Functional TE-OCT images can be reconstructed based on the displacement detecting the optical absorbers, in addition to the conventional OCT images.

In previous work, Kim et al. demonstrated the study of thermo-elastic deformation using a longer duration $(>50 \mathrm{~ms})$ pulse laser and a slower OCT engine [36]. The study focused on the surface displacement and did not provide image information, as shown in Fig. 3. Blatter et al. demonstrated the study of extracting displacement within a single spectral sweep using OCT, which was also focused on a single interface [37]. Song et al. used short laser pulses as an excitation source to study acoustic wave propagation [38]. Spectroscopic OCT (S-OCT) has been applied for imaging differential absorption in tissue [39]. TE-OCT does not trade spatial for spectral information content the way S-OCT does, and can measure absorption outside the OCT band, as the pulsed laser wavelength can be freely chosen. TE-OCT exploits a similar contrast mechanism as photoacoustic imaging, which "hears" the sound wave induced by the pulse laser absorption and can be combined with ultrasonic pulse echo imaging for structural information $[31,40]$. TE-OCT extracts the structural and composition information from a single all-optical measurement by a phase-sensitive $\mathrm{MHz}$ OCT, directly "seeing" the thermo-elastic deformation.

The imaging speed of our current prototype is limited by the repetition frequency of the pulsed laser. A faster pulsed laser can potentially be used to achieve real-time volumetric TE-OCT imaging [41]. Our phantom studies focus on the optical absorption and optical parameter extraction. Other physical parameters can also be extracted by studying the rapid expansion and the contraction. However, this will require a much faster phase-sensitive OCT system, together with a threedimensional numerical modeling $[29,30]$. The temperature 
increase at every pulse is in the order of a millikelvin, which is similar to photoacoustic imaging [31]. It is necessary to focus the short-pulse laser beam to achieve a similar or smaller beam spot than the OCT beam. Such focusing will significantly reduce the short-pulse laser power requirement and avoid any thermal buildup by repeated irradiation of tissue.

We believe that TE-OCT can potentially be used for diseased tissue identification requiring the integration of a short-pulse laser to the current OCT platform. TE-OCT may potentially be used for lipid-rich plaque detection, where lipid has stronger optical absorption at 1200 and $1700 \mathrm{~nm}$ than other tissues [42]. The short deformation accumulation time and the laser-based excitation make it possible to apply TE-OCT for endoscopic imaging at an A-line rate of 50$100 \mathrm{kHz}$, depending on the time required to capture the transient. Catheter imaging probes are feasible using a doublecladding fiber design [43] and an optimized focusing component [44]. Stacked structures such as multiple absorbing layers will interact through the elasticity of the tissue inducing difficulties in distinguishing. We will take elastic properties into account in the follow-up studies to investigate more complex structures such as multiple absorbing layers.

Funding. Nederlandse Organisatie voor Wetenschappelijk Onderzoek (NWO) (104003006); Deutsche Forschungsgemeinschaft (DFG) (DFG-HU 1006/6); H2020 European Research Council (ERC) (ERC CoG ENCOMOLE-2i, 646669).

Acknowledgment. The authors acknowledge Geert Springeling for his contribution to the probe manufacturing and phantom preparation. T. Wang and M. Wu acknowledge support by the Netherlands Organization for Scientific Research (NWO) ZonMW. T. Pfeiffer, W. Wieser, and R. Huber acknowledge support by the German Research Foundation and the European Union.

†These authors contributed equally to this Letter.

\section{REFERENCES}

1. D. Huang, E. A. Swanson, C. P. Lin, J. S. Schuman, W. G. Stinson, W. Chang, M. R. Hee, T. Flotte, K. Gregory, C. A. Puliafito, and J. G. Fujimoto, Science 254, 1178 (1991).

2. J. M. Schmitt, IEEE J. Sel. Top. Quantum 5, 1205 (1999).

3. R. Leitgeb, C. K. Hitzenberger, and A. F. Fercher, Opt. Express 11, 889 (2003)

4. S. H. Yun, G. J. Tearney, J. F. de Boer, N. Iftimia, and B. E. Bouma, Opt. Express 11, 2953 (2003).

5. E. A. Swanson, J. A. Izatt, M. R. Hee, D. Huang, C. P. Lin, J. S. Schuman, C. A. Puliafito, and J. G. Fujimoto, Opt. Lett. 18, 1864 (1993).

6. A. M. Zysk, F. T. Nguyen, A. L. Oldenburg, D. L. Marks, and S. A. Boppart, J. Biomed. Opt. 12, 051403 (2007).

7. A. F. Fercher, C. K. Hitzenberger, W. Drexler, G. Kamp, and H. Sattmann, Am. J. Ophthalmol. 116, 113 (1993).

8. M. Wojtkowski, R. Leitgeb, A. Kowalczyk, T. Bajraszewski, and A. F. Fercher, J. Biomed. Opt. 7, 457 (2002).

9. C. A. Puliafito, M. R. Hee, C. P. Lin, E. Reichel, J. S. Schuman, J. S. Duker, J. A. Izatt, E. A. Swanson, and J. G. Fujimoto, Ophthalmology 102, 217 (1995).

10. J. Welzel, E. Lankenau, R. Birngruber, and R. Engelhardt, J. Am. Acad. Dermatol. 37, 958 (1997).

11. B. E. Bouma, G. J. Tearney, C. C. Compton, and N. S. Nishioka, Gastrointest. Endosc. 51, 467 (2000).
12. J. Rogowska, N. A. Patel, J. G. Fujimoto, and M. E. Brezinski, Heart 90, 556 (2004).

13. X. Liang, S. G. Adie, R. John, and S. A. Boppart, Opt. Express 18 , 14183 (2010).

14. B. F. Kennedy, K. M. Kennedy, and D. D. Sampson, IEEE J. Sel. Top. Quantum 20, 272 (2014).

15. J. F. de Boer, T. E. Milner, M. J. van Gemert, and J. S. Nelson, Opt. Lett. 22, 934 (1997).

16. G. van Soest, T. Goderie, E. Regar, S. Koljenovic, G. L. van Leenders, N. Gonzalo, S. van Noorden, T. Okamura, B. E. Bouma, G. J. Tearney, J. W. Oosterhuis, P. W. Serruys, and A. F. van der Steen, J. Biomed. Opt. 15, 011105 (2010).

17. T. Ma, W. J. Qi, R. Li, Q. F. Zhou, K. K. Shung, and Z. P. Chen, in IEEE International Ultrasonics Symposium (2013), p. 1154.

18. B. F. Kennedy, S. H. Koh, R. A. McLaughlin, K. M. Kennedy, P. R. T. Munro, and D. D. Sampson, Biomed. Opt. Express 3, 1865 (2012).

19. M. Singh, C. Wu, C. H. Liu, J. S. Li, A. Schill, A. Nair, and K. V. Larin, Opt. Lett. 40, 2588 (2015).

20. M. C. Skala, M. J. Crow, A. Wax, and J. A. Izatt, Nano Lett. 8, 3461 (2008).

21. C. Zhou, T. H. Tsai, D. C. Adler, H. C. Lee, D. W. Cohen, A. Mondelblatt, Y. Wang, J. L. Connolly, and J. G. Fujimoto, Opt. Lett. 35, 700 (2010).

22. J. M. Tucker-Schwartz, T. A. Meyer, C. A. Patil, C. L. Duvall, and M. C. Skala, Biomed. Opt. Express 3, 2881 (2012).

23. D. Hillmann, H. Spahr, C. Pfaffle, H. Sudkamp, G. Franke, and G. Huttmann, Proc. Natl. Acad. Sci. USA 113, 13138 (2016).

24. B. Yin, R. V. Kuranov, A. B. McElroy, S. Kazmi, A. K. Dunn, T. Q. Duong, and T. E. Milner, J. Biomed. Opt. 18, 56005 (2013).

25. J. M. Tucker-Schwartz, M. Lapierre-Landry, C. A. Patil, and M. C. Skala, Biomed. Opt. Express 6, 2268 (2015).

26. B. Soroushian, W. M. Whelan, and M. C. Kolios, J. Biomed. Opt. 15, 065002 (2010).

27. B. P. Payne, V. Venugopalan, B. B. Mikic, and N. S. Nishioka, J. Biomed. Opt. 8, 264 (2003).

28. I. Itzkan, D. Albagli, M. L. Dark, L. T. Perelman, C. Vonrosenberg, and M. S. Feld, Proc. Natl. Acad. Sci. USA 92, 1960 (1995).

29. S. R. Aglyamov, S. Wang, S. Y. Emelianov, and K. V. Larin, Proc. SPIE 9710, 971010 (2016).

30. D. Albagli, M. Dark, C. von Rosenberg, L. Perelman, I. Itzkan, and M. S. Feld, Med. Phys. 21, 1323 (1994).

31. J. Xia, J. Yao, and L. V. Wang, Electromagnetic Waves 147, 1 (2014).

32. D. C. Adler, W. Wieser, F. Trepanier, J. M. Schmitt, and R. A. Huber, Opt. Express 19, 20930 (2011).

33. R. Huber, M. Wojtkowski, and J. G. Fujimoto, Opt. Express 14, 3225 (2006).

34. W. Wieser, W. Draxinger, T. Klein, S. Karpf, T. Pfeiffer, and R. Huber, Biomed. Opt. Express 5, 2963 (2014).

35. A. Kharine, S. Manohar, R. Seeton, R. G. M. Kolkman, R. A. Bolt, W. Steenbergen, and F. F. M. de Mul, Phys. Med. Biol. 48, 357 (2003).

36. J. Kim, H. W. Kang, J. Oh, and T. E. Milner, Biomed. Opt. Express $\mathbf{5}$ 474 (2014).

37. C. Blatter, B. Grajciar, P. Zou, W. Wieser, A. J. Verhoef, R. Huber, and R. A. Leitgeb, Opt. Lett. 37, 4368 (2012).

38. S. Z. Song, B. Y. Hsieh, W. Wei, T. Shen, I. Pelivanov, M. O'Donnell, and R. K. K. Wang, Proc. SPIE 9697, 969710 (2016).

39. R. Leitgeb, M. Wojtkowski, A. Kowalczyk, C. K. Hitzenberger, M. Sticker, and A. F. Fercher, Opt. Lett. 25, 820 (2000).

40. L. V. Wang and S. Hu, Science 335, 1458 (2012).

41. M. Wu, G. Springeling, M. Lovrak, F. Mastik, S. Iskander-Rizk, T. Wang, H. M. M. van Beusekom, A. F. W. van der Steen, and G. Van Soest, Biomed. Opt. Express 8, 943 (2017).

42. K. Jansen, M. Wu, A. F. van der Steen, and G. van Soest, Photoacoustics 2, 12 (2014).

43. D. Lorenser, B. C. Quirk, M. Auger, W. J. Madore, R. W. Kirk, N. Godbout, D. D. Sampson, C. Boudoux, and R. A. McLaughlin, Opt. Lett. 38, 266 (2013)

44. A. M. Fard, P. Vacas-Jacques, E. Hamidi, H. Wang, R. W. Carruth, J. A. Gardecki, and G. J. Tearney, Opt. Express 21, 30849 (2013). 